\title{
Prison et résistances politiques. Le grondement de la bataille
}

Philippe Artières, Pierre Lascoumes et Grégory Salle

\section{(2) OpenEdition \\ 1 Journals}

\section{Édition électronique}

URL : http://journals.openedition.org/conflits/1555

DOI : $10.4000 /$ conflits. 1555

ISSN : $1777-5345$

Éditeur :

CCLS - Centre d'études sur les conflits lilberté et sécurité, L'Harmattan

\section{Édition imprimée}

Date de publication : 1 septembre 2004

Pagination : 5-14

ISBN : 2-7475-7583-7

ISSN : 1157-996X

\section{Référence électronique}

Philippe Artières, Pierre Lascoumes et Grégory Salle, «Prison et résistances politiques. Le grondement de la bataille», Cultures \& Conflits [En ligne], 55 | automne 2004, mis en ligne le 08 janvier 2010, consulté le 30 mars 2021. URL : http://journals.openedition.org/conflits/1555 ; DOI : https://doi.org/ 10.4000/conflits. 1555

Ce document a été généré automatiquement le 30 mars 2021.

Creative Commons License 


\title{
Prison et résistances politiques. Le grondement de la bataille
}

\author{
Philippe Artières, Pierre Lascoumes et Grégory Salle
}

1 La prison fonctionne comme un trou de mémoire. Dans le sillage de Surveiller et Punir ${ }^{1}$, et parfois en démentant ou en nuançant certaines de ses thèses, les historiens ont produit d'importants travaux sur la naissance et les deux siècles d'existence de l'institution pénitentiaire moderne comme mode de pénalité ${ }^{2}$. Appuyant l'idée foucaldienne selon laquelle la critique de la prison est consubstantielle de son existence et qu'elle est marquée par les mêmes répétitions, ils ont montré combien sont récurrentes les dénonciations des effets néfastes de l'incarcération : "Dès 1791, tout a été dit, ou presque, sur la prison, ou plutôt contre la prison $»^{3}$. Mais rien n'y fait : à chaque crise, le "législateur $»^{4}$ redécouvre la prison et la puissance de ses logiques. Il se livre ostensiblement à une prise de conscience "authentique», comme s'il prenait réellement la mesure des dommages sociaux générés par l'enfermement. Le poids et les effets de l'architecture, la misère des conditions d'existence, l'écrasement du temps et de l'espace sont inlassablement rappelés. La nature et les modalités de l'exercice de ce pouvoir implacable ont maintes fois été décrites, détaillées, démasquées. L'amnésie est sans doute l'une des conditions de régénération de la solution carcérale («l'extrême solidité de la prison, cette mince invention décriée pourtant dès sa naissance $\left.»^{5}\right)$, dont la force motrice tient paradoxalement au constat récurrent de son échec, duquel elle tire prétexte pour reconduire sa nécessité. Tout se passe alors comme si cette institution n'avait pas d'histoire.

Plus grand encore demeure le silence sur ce qui se passe ou s'est passé d'inhabituel derrière ces hauts murs. Par définition, l'emprisonnement fonctionne selon le principe d'une soustraction physique et symbolique de l'individu du cours social ordinaire et de la visibilité publique. Reclus et atomisé, il se trouve destitué de son droit à l'action et à la parole. Il est ainsi soumis à l'ordre de ce que Jacques Rancière définit comme la "police »: un «ordre du visible et du dicible qui fait que telle activité est visible et que telle autre ne l'est pas, que telle parole est entendue comme du discours et telle autre comme $d u$ bruit $»^{6}$. La possibilité d'amorcer des mouvements collectifs est réduite à l'extrême, et leur actualisation constitue une menace pour l'ordre carcéral, qu'il convient d'écraser, 
et/ou de rendre inaudible. En ce qui concerne les sciences sociales, l'enquête ethnographique a fait voir qu'une enceinte carcérale n'est pas un univers immobile et froid, mais qu'elle est au contraire un espace en tension perpétuelle dans lequel se jouent de multiples manœuvres et transactions pour maintenir un équilibre précaire entre « guerre » et " paix $»^{7}$. Une série de témoignages publiés par des détenus souligne cet autre plan de l'activité pénitentiaire, cette vie quotidienne et informelle sous tension $^{8}$. Les archives laissent également parfois transpirer cet ordinaire extraordinaire, où un incident peut devenir événement qui exige des écritures. Pour le $\mathrm{XIX}^{\mathrm{ème}}$ siècle, les archives du bagne de Cayenne sont particulièrement riches en ce domaine et donnent à voir la fréquence et l'intensité de ces grondements, inscrits au centre de ces existences coupables 9 .

3 Du point de vue de l'opposition politique hostile à l'ordre établi, la mise en détention marque communément la fin du parcours, un coup d'arrêt dans la lutte, quand elle ne consacre pas brutalement la défaite. Or, en diverses circonstances de l'histoire contemporaine, la prison a été retournée. Parce que lieu de répression ou de discipline, elle a pu se muer un moment en un espace de résistance, ou en point d'appui pour une action militante extérieure. Ces épisodes de résistance ont été pour partie étudiés par les historiens du XIX ${ }^{\text {ème }}$ siècle. Des travaux, initiés notamment par la Société d'Histoire de la Révolution de 1848 , se sont intéressés à la vie en détention d'un certain nombre de figures ou de mouvements politiques victimes de répression ; il s'agissait en définitive de caractériser cette répression et d'en mesurer les effets sur ses victimes ${ }^{10}$. L'emprisonnement était donc étudié comme moment dans une trajectoire politique ${ }^{11}$. Plusieurs travaux ont ainsi porté sur l'activité politique de militants lors de leur incarcération. Ils s'inscrivaient dans la perspective d'une histoire de la prison politique notamment développée par Jean-Claude Vimont ${ }^{12}$. La visée de ces recherches n'était pas tant d'examiner les révoltes dont les prisons du XIX ${ }^{\text {ème }}$ siècle avaient pu être le théâtre, que d'analyser comment pour chaque groupe de politiques entrant en détention, l'emprisonnement s'était accompli et avait été vécu ${ }^{13}$.

4 La prison a été constituée, dans des contextes particuliers, en sujet et en objet de lutte collective, et non pas seulement individuelle, devons-nous immédiatement ajouter. On sait en effet depuis les travaux d'Erving Goffman ${ }^{14}$ que les institutions totales sont le lieu d'une multitude d' "adaptations" et de dérangements opérés par ceux qui y séjournent. Ces révoltes individuelles qui prennent parfois dans les prisons la forme d'actes d'automutilation corporelle et de suicides sont des actions contre l'institution carcérale. On doit cependant se demander si ces actes individuels de révolte ne sont pas le produit de l'institution, non seulement parce que la prison les génère, mais aussi parce qu'ils participeraient de son bon fonctionnement social. Aussi, voudrions-nous dans ce numéro explorer quelques uns de ces moments singuliers où l'incarcération a été détournée et pensée collectivement par les prisonniers comme un levier, un instrument d'engagement et de mobilisation politiques. Il s'agit d'étudier sous cet angle les articulations entre l'intérieur et l'extérieur. En effet, si les rapports entre les politiques pénitentiaires et le politique comme événement commencent à être mieux connus $^{15}$, ces épisodes de politisation de et dans la prison restent largement dans l'ombre. Quelques travaux de recherche ont certes été menés sur la répression politique et l'usage fait de l'enfermement par les gouvernants, mais rares sont les travaux qui cherchent à décrire précisément et à caractériser les mouvements de résistance qui se développent en détention ou autour d'elle ${ }^{16}$. En d'autres termes, les luttes autour de la prison n'ont guère été travaillées comme une scène à part entière du 
politique, où se produisent, s'importent et s'adaptent des modes d'action génériques (c'est-à-dire initialement valables pour le "dehors») et spécifiques. Si le mot est polysémique, c'est en un sens précis que ces résistances peuvent être qualifiées de " politiques », dès lors qu'elles troublent les partages établis par la police : «La politique existe lorsque l'ordre naturel de la domination est interrompu par l'institution d'une part des sans-part (...) La politique est d'abord le conflit sur l'existence d'une scène commune, sur l'existence et la qualité de ceux qui sont présents ${ }^{17}$.

Dans une perspective généalogique, donc critique, ce numéro propose d'aborder ces luttes à partir d'un ensemble de cas étrangers et français au cours du siècle qui vient de se clore. Il ne s'agit pas seulement d'exhumer ces moments de résistance pour euxmêmes, dans un simple souci d'érudition, mais aussi de contribuer à rendre compte des modalités diverses de lutte qui y furent à l'œuvre, la multiplicité des instruments de combat qu'elles ont su inventer et des actions qu'elles purent développer dans chaque contexte géographique et historique particulier. Il s'agit ici d'abord de restituer des singularités sans céder trop hâtivement à la tentation de les subsumer sous des modèles généraux. C'est aussi, dans un premier temps, une manière d'éviter l' «illusion étiologique " pointée par Michel Dobry : la tendance à rabattre l'explication des crises sur leurs origines, leurs causes, leurs conditions d'émergence ${ }^{18}$. Cette réduction se fait notamment au détriment d'une description potentiellement riche des dynamiques des crises elles-mêmes, dont le cours est par nature incertain et ne saurait se résumer en quelques déterminations structurelles. La présentation de ces cas d'étude, divers autant par leur genèse que par leurs implications et leurs effets, fournit néanmoins une palette d'expériences situées qui peuvent servir de matériaux pour engager des montées en généralité et élaborer des hypothèses théoriques sur ce que veut dire « résister » à la discipline carcérale. Les travaux de Foucault sont dans cette perspective d'une grande utilité ; le concept de « résistance » qu'il propose permet d'esquisser une véritable typologie de ces processus. Il y a en effet des résistances individuelles, et d'autres collectives; il y a les résistances comme affrontements à l'intérieur de jeux de pouvoir bien rôdés, et les résistances qui refusent le jeu lui-même ${ }^{19}$.

6 Sous ce rapport, ces quelques éléments visent à donner consistance à cette analyse du pouvoir qui cherche à le saisir par la résistance. Appréhender le pouvoir sous l'angle de ces pratiques, c'est concevoir celles-ci comme des opérateurs de description, d'explicitation et de clarification, des «catalyseurs chimiques» qui permettent de cerner plus précisément les mécanismes et points d'appui par lesquels transitent différents modes d'exercice du pouvoir ${ }^{20}$. Les violences ainsi mises à jour s'avèrent aussi bien directes que diffuses, elles déploient leurs forces tant sur les corps que sur les représentations, elles fonctionnent à l'injonction mais aussi au non-dit. Au regard de cette complexité, il semble par exemple un peu court de dire que l'ordre carcéral s'alimente exclusivement du secret et de la dissimulation, par opposition à une visibilité unitaire et forcément bénéfique. La question de la publicité constitue autant un problème qu'une solution, et ce qui est montré ne mérite pas moins l'attention que ce qui est caché. L'analyse des rapports entre prison et publicité fait face à des régimes du visible ambivalents qui sont toujours non des états, mais des champs de force: "Depuis le XVIII ${ }^{\text {èe }}$ siècle, en effet, la visibilité sociale a fonctionné comme principe de limitation des pratiques de gouvernement - la publicité étant le moyen d'assurer la protection des individus -, mais également comme principe d'une technologie gouvernementale rationnelle - la publicité devenant alors le moyen de maximiser l'efficacité du pouvoir au moindre coût $»^{21}$. 
7 Les pratiques de résistance ${ }^{22}$ s'opposent-elles ou, à l'inverse, s'articulent-elles aux pratiques du pouvoir? Foucault s'est très tôt confronté à ce double paradoxe. Tout d'abord, c'est dans les espaces sociaux où la contrainte est supposée la plus forte, où l'exercice du pouvoir semble le plus contraignant que Foucault est allé rechercher les indicateurs d'une résistance possible. Dans sa préface au livre de Bernard Cuau sur l'affaire Mirval, Foucault invoque la nécessaire résistance de «la vérité " face aux violences institutionnelles ${ }^{23}$. Ensuite, il ne fait pas des oppositions, des critiques, des contestations ou des révoltes violentes une entrave à l'exercice de la domination; au contraire, il inverse le regard à leur endroit et incite à les envisager comme des pratiques qui fonctionnent, indépendamment de leurs intentions premières, comme des facteurs contribuant à sa consolidation. Il déclare en 1977 à propos du panoptique : "Il faut, cela étant, analyser l'ensemble des résistances au panoptique en termes de tactique et de stratégie, en se disant que chaque offensive d'un côté sert de point d'appui à une contreoffensive de l'autre côté $»^{24}$. C'est en 1973, dans le cadre de sa réflexion sur "les illégalismes populaires » et la constitution de la société punitive que la notion de résistance prend chez Foucault un sens spécifique. Le resserrement des liens de propriété, ou plutôt le nouveau statut de la propriété terrienne et sa nouvelle exploitation transforme en délits nombre d'illégalismes installés. Foucault voit dans cette nouvelle gestion des illégalismes la pénalisation de résistances populaires aux nouvelles législations - en particulier les codes forestiers du début du XIX ${ }^{\text {ème }}$ siècle $^{25}$. La même conception est reprise à propos de l'anti-psychiatrie en 1974 et de la résistance du malade à l'interprétation médicale de ses discours et de ses comportements ${ }^{26}$. Plus tard, il présente la médicalisation de sociétés occidentales comme une tentative de réponse aux nouvelles formes de résistance induites par le capitalisme montant: "Lorsqu'on a eu peur aussi que des mouvements populaires de résistance, ou d'inertie, ou de révolte viennent bouleverser tout cet ordre capitaliste en train de naitre, alors il a fallu une surveillance précise et concrète sur tous les individus, et je crois que la médicalisation (...) est liée à cela ${ }^{27}$.

C'est dans le chapitre intitulé "Méthode" de La volonté de savoir que Foucault a présenté de la façon la plus synthétique son cadre d'analyse du pouvoir ${ }^{28}$. Son hypothèse la plus originale renverse le regard traditionnel et prend à rebours les théories qui depuis quatre siècles mettent au centre de leurs analyses la puissance souveraine, puis celle de l'Etat. La loi ou une domination perçue comme globale et unitaire ne sont pour lui que des "formes terminales", c'est-à-dire plutôt des résultantes que des causes ${ }^{29}$. Comprendre les phénomènes de pouvoir suppose de saisir "la multiplicité des rapports de force qui sont immanents au domaine où ils s'exercent, et sont constitutifs de son organisation ${ }^{30}$. Ainsi les lieux de friction, les affrontements, les conflits sont des phénomènes constants qui contribuent autant à maintenir l'identité et la cohérence des institutions qu'à les déstabiliser et les mettre en cause. "La grille d'intelligibilité $d u$ social» n'est pas à rechercher dans "l'existence première d'un point central, dans un foyer unique de souveraineté d'où rayonneraient des formes des forces dérivées et descendantes"; il est au contraire à repérer dans le "le socle mouvant des rapports de force qui induisent sans cesse, par leur inégalité, des états de pouvoir, mais toujours locaux et instables». Le mouvement est cependant symétrique et chaque "avancée du pouvoir provoque en retour un mouvement de résistance $»^{31}$. En reprenant une terminologie plus contemporaine, on peut dire que pour Foucault les résistances participent de l'institutionnalisation des pouvoirs à l'œuvre. Mais cela ne veut pas dire qu'elle les légitiment: elles contribuent à en redéfinir les frontières toujours mouvantes, à 
déterminer à nouveau le cadre des affrontements, les contours des arènes sociopolitiques. La prison est ainsi un lieu exemplaire de ces hétérotopies, ces « espaces autres » dotés d'une assez grande stabilité malgré la virulence des attaques dont ils sont régulièrement l'objet ${ }^{32}$.

En outre, là où il y a pouvoir, il y a résistance. Celle-ci n'est jamais dans un rapport d'extériorité, en raison du caractère profondément relationnel des rapports de pouvoir : "Ils ne peuvent exister qu'en fonction d'une multiplicité de points de résistance: ceux-ci jouent, dans les relations de pouvoir, le rôle d'adversaire, de cible, d'appui, de saillie pour une prise $»^{33}$. Les points de résistance sont omniprésents, ce qui ne veut pas dire opérants. Il ne faut pas, en tous cas, les considérer comme " un contrecoup, la marque en creux (...) un envers finalement toujours passif, voué à l'indéfinie défaite (...) Elles sont l'autre terme, dans les relations de pouvoir ; elle s'y inscrivent comme l'irréductible vis à vis $»^{34}$. C'est pourquoi l'analyse des résistances, de leurs modalités d'existence et de leur distribution, est partie intégrante des relations de pouvoir. Mais ce travail présente des difficultés particulières en raison du caractère particulièrement mobile et transitoire de ces dynamiques, et parce que "l'essaimage des points de résistance traverse les stratifications sociales et les unités individuelles $»^{35}$. Malgré ces obstacles, cette entreprise de repérage et de traçage est essentielle, car elle permet de repérer les lignes de fragilité et de fracture et donc les points possibles de changements structuraux : «C'est sans doute le codage stratégique de ces points de résistance qui rend possible une révolution, un peu comme l'Etat repose sur l'intégration institutionnelle des rapports de pouvoir ». Foucault fait ici le lien avec sa réflexion générale sur la gouvernementalité et sa rupture avec les conceptions transcendantes : il est temps de se débarrasser des figures du Prince et du Léviathan, pour aborder les stratégies immanentes qui tissent les rapports de force. Dans un de ses derniers textes (1984) la notion de résistance prend une place centrale dans les processus de changement: «Je n'ai pas voulu dire que nous étions toujours piégés, mais, au contraire, que nous sommes toujours libres. Enfin, bref, qu'il y a toujours la possibilité de transformer les choses (...) s'il n'y avait pas de résistance, il n'y aurait pas de rapports de pouvoir. Parce que tout serait simplement une question d'obéissance. Dès l'instant où l'individu est en situation de ne pas faire ce qu'il veut, il doit utiliser les rapports de pouvoir. La résistance vient donc en premier, et elle reste supérieure à toutes les forces du processus; elle oblige, sous son effet, les rapports de pouvoir à changer. Je considère donc que le terme de "résistance » est le mot le plus important, le mot-clef de cette dynamique» ${ }^{36}$.

10 Mus par des préoccupations et des problématiques propres, par des auteurs provenant de différentes disciplines, les textes présentés dans ce numéro sont volontairement hétérogènes dans leur forme comme leur contenu. A la charnière du XIX ${ }^{\text {ème }}$ et du XX ${ }^{\text {ème }}$ siècle, l'histoire de Vera Figner illustre le cas de la prise de parole comme pratique de résistance, de l'autobiographie comme dénonciation de la répression politique dans le système pénitentiaire de la Russie tsariste (P. Artières). La prison franquiste donne à voir les enjeux de la catégorisation de la dissidence politique, et la primauté d'un enfermement qui vise à plier et transformer les détenus, et en fin de compte à dissoudre leur identité (R. Vinyes). Plus tard, la brèche ouverte par l'après-68 donne naissance, dans de nombreux pays, à des entreprises critiques et à des actions militantes radicales. En France, le Groupe d'Information sur les Prisons (GIP) se fixe le projet de faire savoir publiquement et de dénoncer l'exercice d'un pouvoir «intolérable» (G. Salle). C'est une manière de lutter contre l'opacité qui semble inhérente au système carcéral, à ce moteur du secret mis en relief par un épisode méconnu, l' «affaire Mirval » au milieu des années 1970 (F. Boullant). Durant la même 
décennie, dans le contexte de la guérilla urbaine en Allemagne, le cas de la "réclusion révolutionnaire » expérimentée par les détenus de la Rote Armee Fraktion a constitué la prison comme une véritable arène de conflit entre le groupe révolutionnaire et l'Etat, offerte au jugement des tiers (D. Linhardt). Le cas des Black Panthers aux Etats-Unis est abordé au travers d'un document, un texte militant de janvier 1970. Près de nous enfin, un entretien avec Paolo Persichetti, intellectuel italien extradé par un effet de rémanence des "années de plomb" qui dépeint la situation actuelle des droits politiques dans les prisons italiennes, fait apparaître ces épisodes de lutte collective comme des fragments révolus du passé (A. Brossat).

\section{NOTES}

1. Foucault M., Surveiller et Punir. Naissance de la prison, Paris, Gallimard, 1975.

2. Perrot M., Les ombres de l'histoire. Crime et châtiment au XIXème siècle, Paris, Flammarion, 2001 ; Petit J.G., Ces peines obscures. La prison pénale en France 1780-1875, Paris, Fayard, 1990 ; O’Brien P., Correction ou châtiment. Histoire des prisons en France au XIXème siècle, Paris, PUF, 1988.

3. Petit J.G., op. cit., p. 54.

4. C'est par raccourci qu'on évoque cette entité fictive et désincarnée de la théorie libérale, qui recouvre l'existence de communautés savantes en concurrence pour l'imposition de la pénalité légitime. Ces «communautés épistémiques» sont prises dans des rapports changeants, entre intimité et conflictualité, avec les représentants élus (Lascoumes P., Poncela P., Réformer le Code pénal. Mais où est passé l'architecte ?, Paris, PUF, 1998).

5. Foucault M., Surveiller et Punir, op. cit., p. XX.

6. Rancière J., La Mésentente. Politique et philosophie, Paris, Galilée, p. 52.

7. Chauvenet A., "Guerre et paix en prison », Les cahiers de la sécurité intérieure, vol. 31, 1998, pp. 91-100. Parmi les travaux récents d'inspiration ethnographique, voir notamment Chantraine G., Au-delà des murs. Trajectoires et expériences en maison d'arrêt, Paris, PUF, 2004 ; Le Caisne L., Prison. Une ethnologue en centrale, Paris, Odile Jacob, 2000 ; Rostaing C., La relation carcérale. Identités et rapports sociaux dans les prisons de femmes, Paris, PUF, 1997.

8. Citons entre autres les écrits d'Albertine Sarrazin, les ouvrages de Serge Livrozet et Jacques Lesage de la Haye pour les années 1970, ou encore le récent texte de Laurent Jacqua, La Guillotine carcérale. Silence, on meurt, Paris, Nautilus, 2003.

9. Nous renvoyons ici pour exemple au récit d'un prisonnier de droit commun, Camille Honoré Petitjean, qui dans son autobiographie rapporte notamment une révolte en 1900 sur le bateau qui conduisait les bagnards en Guyane (A ce sujet, voir Artières P., Le livre des vies coupables, Paris, Albin-Michel, 2000, pp. 315-356).

10. Voir en particulier le volume de la Société d'Histoire de la Révolution de 1848 et des Révolutions du XIXème siècle, Répression et prisons politiques en France et en Europe au XIXème siècle, Paris, Créaphis, 1990.

11. Voir par exemple Rude F., «Les insurgés d'avril 1834 à Clairvaux et à Doullens » et Huard R., «Une détention et sa chronique : les communards de la maison centrale de Nîmes (1871-1874) in Répression et prison politiques, op. cit., respectivement pp. 205-221 et pp. 223-235.

12. Vimont J-C., La Prison politique en France (XVIIİ̀me-XXème siècle), Paris, Anthropos, 1993. 
13. Pour le XIXème siècle, peu de sources sont disponibles pour écrire l'histoire des révoltes. Voir sur ce point : Artières P., «La prison en procès. Les Mutins de Nancy (1972) » in Vingtième siècle. Revue d'histoire, $n^{\circ} 70$, avril-juin 2001, pp. 57-70.

14. Goffman E., Asiles. Etudes sur la condition sociale des malades mentaux, Paris, Minuit, 1990 [1968]. 15. Voir les contributions réunies dans Artières P., Lascoumes P. (dir.), Gouverner, enfermer. La prison, un modèle indépassable?, Paris, Presses de Sciences Po, 2004.

16. Les repères bibliographiques établis par Philippe Combessie démontrent cette lacune (Combessie P., Sociologie de la prison, Paris, La Découverte, 2001).

17. Rancière J., La Mésentente. Politique et philosophie, op. cit., pp. 31 et 49.

18. Dobry M., Sociologie des crises politiques, Paris, FNSP, 1992, notamment pp. 48 et s.

19. Foucault M., «La philosophie analytique de la politique », Dits et Ecrits, t. 2, Paris, Gallimard, 1994, n²32, p. 543.

20. Dreyfus H., Rabinow P., Michel Foucault. Un parcours philosophique, Paris, Gallimard, 1984 (en particulier le chapitre X, «Questions et réponses », pp. 293-321).

21. Senellart M., Les arts de gouverner : du régiment médiéval au concept de gouvernement, Paris, Seuil, 1995, p. 283. Rappelons l'ambivalence inhérente au panoptique benthamien, conçu comme le vecteur parachevé à la fois d'une transparence et d'une surveillance totales.

22. Longtemps utilisée par Foucault au sens d'un obstacle épistémologique, la notion de résistance prend un tout autre contenu à partir de l'expérience du GIP et de sa réflexion sur les illégalismes dans ses cours de 1973. Sur la première conception, voir Dits et écrits, t.1, Paris, Gallimard, 1994, pp. 149, 267 ; t.2, op. cit., pp. 54-55. Sur la seconde, « La société punitive ", Dits et écrits, t. 2, op. cit., n¹31, pp. 467 et s.

23. Foucault M., « Une mort inacceptable », Dits et écrits, t.3, Paris, Gallimard, 1994, pp. 7-9. Lire aussi dans ce volume la contribution de F. Boullant.

24. Foucault M., « L'œil du pouvoir », Dits et écrits, t.2, op. cit., n¹95, p. 206.

25. «La société punitive », op. cit., p. 467.

26. Foucault M., « Le pouvoir psychiatrique », Dits et écrits, t. 2, op. cit., n 163, p. 684.

27. Foucault M., « Le pouvoir, une bête magnifique », Dits et écrits, t. 3, op. cit., n²12, p. 374.

28. Foucault M., La volonté de savoir, Paris, Gallimard (collection «Tel»), 1976, pp. 121-135. Sauf mention contraire, les citations qui suivent en sont issues.

29. Les réticences de Foucault à traiter tel quel de l'Etat procèdent de l'idée que partir de l'Etat et de la souveraineté serait une mauvaise manière de poser les problèmes. Deleuze a insisté sur le fait que la mise en évidence du diagramme disciplinaire s'attache à restituer la prolifération des rapports de pouvoir qui, s'ils investissent l'Etat, le débordent pour se loger dans une infinité de lieux sociaux. En récusant la conception de l'Etat comme source, en concevant l'Etat comme une «forme terminale», il s'agit de penser plutôt les processus d'étatisation: voir Deleuze G., Foucault, Paris, Minuit, 1986, notamment p. 82.

30. Foucault M., La volonté de savoir, op. cit.

31. «Le pouvoir, une bête magnifique », op. cit., p. 382.

32. Foucault M., « Des espaces autres », Dits et écrits, op. cit., t. 4, n 360, p. 752 et s.

33. Foucault M., La volonté de savoir, op. cit.

34. Foucault M., ibid.

35. Ibid.

36. Foucault M., «Sexe, pouvoir et la politique de l'identité », Dits et écrits, op. cit., t. 4, n³58, p. 741. 
INDEX

Mots-clés : Introduction, prison, surveillance, résistances politiques

\section{AUTEURS}

\section{PHILIPPE ARTIÈRES}

Philippe Artières est chargé de recherche au CNRS (LAHIC-UMR2558). Il a publié avec Pierre Lascoumes (dir.), Gouverner, enfermer. La prison, un modèle indépassable? (Paris, Presses de Sciences Po, 2004)

\section{PIERRE LASCOUMES}

Pierre Lascoumes est directeur de recherche CNRS - Cevipof - IEP Paris

\section{GRÉGORY SALLE}

Grégory Salle est doctorant en science politique à l'IEP de Paris. Achève actuellement une thèse, sous la direction de Pierre Lascoumes, portant sur les conditions de la force et de la légitimité de l'institution carcérale à l'épreuve d'événements historiques, à partir de cas d'étude allemand et français 